\title{
Self-care or self-management in palliative survivorship care in Asia: A call for more research
}

\author{
Siew Yim Loh* \\ Faculty of Medicine, University of Malaya. 50630, Kuala Lumpur, Malaysia
}

\begin{abstract}
A rising trend of survivorship and palliative care calls for innovative ways to manage the chronic phases of cancer condition. Self-management support is not a new concept but there are evidences of benefits in developed countries. However, the complementary relationship between self-management and palliative care to enable survivors to live with cancer as a prolonged chronic illness in Asia must be evaluated in terms of appropriateness and timeliness.

This paper highlight some of Asia's strong culture and some lessons learnt from a patient self-management intervention with women living with breast cancer trial conducted longitudinally over 6 years. Although many studies have provided evidence of better support among patients, caregivers and healthcare professionals when self-management interventions are integrated into palliative cancer care, across the care trajectory (from curative care, palliative care, end-of-life care and bereavement phases), tailored interventions are needed to assimilate the culture to improve health outcomes in Asia. A distinction is also made between self-care and self-management.
\end{abstract}

Summary: In resource limited countries, a call to better partnership with (and to support) cancer survivors and their caregivers, across the care trajectory is essential, but adaptations to the methods of deliveries are likely to ensure effectiveness of service. More research is needed in this emerging area so as to optimize care for the rising incidences.

\section{Rising cancer and chronic disease management}

The World Health Organization defines chronic diseases as having one or more of the following characteristics: are permanent, involves residual disability, caused by non-reversible pathological alteration, require special training/ rehabilitation, or require a long period of supervision, observation, or care [1]. Therefore, some cancer like breast and prostate are classified as a form of chronic illness, with challenges being faced over indefinite periods of living with the condition. The Long-Term Medical Conditions Alliance recognized the effect that long-term conditions have on people's emotional and social wellbeing, and the opportunities available to improve a person's quality of life $[2,3]$. The ultimate goal of intervention for cancer as a chronic and palliative condition, is the maintenance of pleasurable - independent living, not cure [4].

\section{Self-care vs self-management concept}

Chronic disease management aim to manage chronic conditions using a systematic care approach that employs multiple treatment modalities [5]. Self-management support is one of the key modalities proposed in the model for chronic condition management, which highlights the three elements of - i) the self-management support, ii) the informed-activated patient and iii) the prepared-proactive team -interacting productively toward functional and clinical outcomes [6]. As a way to support to the health system which is not able to cope with the rising chronic conditions, 'Self-management support' (or the process of making multilevel changes in health care systems, and also in the community) arise - in order to facilitate patient self-management [7]. Unlike acute care, the management of a chronic disease is characterized by the responsibilities patients need to take $[4,8]$.
However, Asian are more familiar with self-care concept. The terms (self-management and self-care concept) have been used interchangeably and confusingly, because the distinction between them is not clear. Both concepts refer to a range of methods and behaviour that enables people to manage their health and illness, leading to a range of positive outcomes [9]. self-care refers to actions and decisions taken independent of interaction with a health professional [10].

Self-management can be referring to, as a subcategory of selfcare (Figure 1) and takes place in the context of a recognized medical condition with a level of health service input [9]. The essential feature here is that, both health professional and patients play collaborative roles as partners for better chronic care management in the three broad tasks of medical, emotional and role tasks, utilising five core skills of, problem solving and decision making, resource utilization, developing effective partnerships with healthcare providers and taking action $[11,12]$. The driver for self-management support resulted from the attempt to enhance the ability of patients to selfmanage the day-to-day challenges of their condition successfully, by linking them to health professionals [13]. These concepts need more work and more research to evaluate how best it can be facilitated to enhance independent living.

*Correspondence to: Siew Yim Loh, Faculty of Medicine, University of Malaya 50630, Kuala Lumpur, Malaysia, E-mail: syloh@um.edu.my

Key words: self-care, self-management, palliative, survivorship, Asia

Received: December 12, 2018; Accepted: December 26, 2018; Published: December 28, 2018 


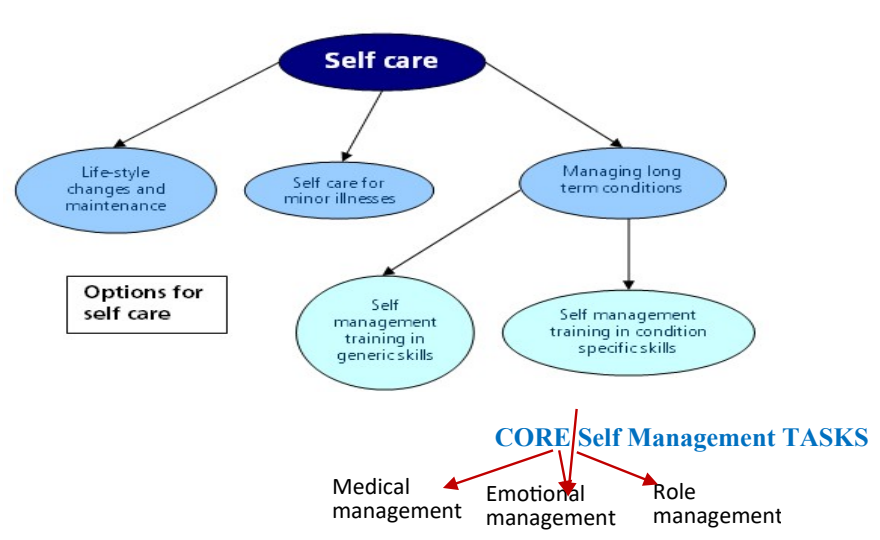

Figure 1. Self-management vs self-care

\section{Cultural adaptations of self-management for Asia}

Independent living in the community suggest several adaptations informed by cultural nuances, stance and beliefs. In a longitudinal trial, evidence of patient's self-management showed evidence of improved QOL although it must be highlighted that the trial was conducted in an urban metropolitan medical centre which may not represent the general multi-ethnic Malaysians with breast cancer [14,15]. This paper attempts to highlight at least seven cultural related issues that needs to be considered when incorporating patient self-management. Unique cultural nuances such as spiritual and language-specific support resource needs (Singh-carlson et al, 2013) must be considered in patient self-management.

First, when a person suffers from chronic illness, the person themselves is not the only one affected by the health condition, and this is especially significant in Asia's extended family system - where the whole family can become a 'unit of patient'.

Second, the self-management needs for persons with chronic conditions can be divided into three types: medical management, role management, and emotional management $[16,17]$. This needs to be slowly introduced to Asian patients, who have been 'nurtured' as passive recipient, to take orders from medical doctors seen by some as 'god'.

Third, the day-to-day care responsibilities by patients and their families are real challenges that need to be dealt with, whereby the resultant challenges/ threats from cancer (suggesting unmet needs with the current medical model) calls for better ways to manage and calls for collaborative partnership with health care providers [6]. Adaptation is also needed in Asia's entrenched medical model governance of health deliveries, where medical doctors leads and are only beginning to learn to collaborate interdisciplinarily with other health professionals $[18,19]$.

Fourth, management of illness aspects is often insufficient in Asia's resource limited scenario where health budget and policies focus on medical doctor and less on healthcare professionals which have been long neglected [6].

Sixth, the emotional wellbeing aspects are needed as part and parcel of the work that people living with chronic palliative conditions, and which must be managed for better outcome and effective living with the condition (Loh psychological Japanese). Emotional distress as a core indicator of patient's wellbeing has been promoted as the sixth vital sign in cancer care [20]. Emotional distress varies in level of intensity, depending on severity of disease and phase of treatment [21]. Evidence suggest that women with (breast) cancer $(n=723,801)$ compared to women in the general population were 37 percent more likely to commit suicide and the elevated risk of suicide persisted long after diagnosis [22].

Seven, throughout Asia, the number of health professionals is way below the norm. As an illustration for comparison, there are 26,000 occupational therapists in Australia's 25 million population but only a handful (less than 2000) occupational therapist in Malaysia's 33 million population [18]. The availability of psychologists is also lacking calling for a better model of group intervention to address the often neglected and unmet emotional-psychosocial issues, and community program with a focus on self-management on wellbeing, rather than focusing on intervening distress may well be the optimal way forward.

Eight, improvements in life expectancy of cancer survivors have also led to a greater emphasis on QOL [23]. QOL assessment can be used in diagnosis, predicting prognosis, assessment, patient monitoring, clinical decision making, communication and treatment [24].

\section{Conclusion}

There is much limited information about patient self-management with Malaysian women, and a critical lack of research and/or a lack of valid, reliable instruments to measure patient-reported outcomes [25]. This paper ends with a strong call for more research in highlighting the lack of health professionals and resources in Asia 's traditional health care deliveries which is incompatible with the rising burden of cancer.

\section{References}

1. Delaware healthcare association (1998) Delaware healthcare association glossary of health care terms and acronyms. Health care professional resources.

2. LTMC (2004) Definition of long-term conditions. About long-term medical conditions.

3. Chew M, Van Der Weyden MB (2003) Chronic illness: The burden and the dream. Med $J$ Aust 179: 229-230. [Crossref]

4. Holman H, Lorig K (2000) Patients as partners in managing chronic disease. Partnership is a prerequisite for effective and efficient health care. BMJ 320: 526-527. [Crossref]

5. Weingarten SR, Henning JM, Badamgarav E, Knight K, Hasselblad V, et al. (2002) Interventions used in disease management programmes for patients with chronic illness--which one's work? Meta-analysis of published reports. BMJ 325: 925. [Crossref]

6. Coleman MT, Newton KS (2005) Supporting self-management in patient with chronic illness. Am Fam Physician 72: 1454-1456. [Crossref]

7. Glasgow RE, Davis CL, Funnell MM, Beck A (2003) Implementing practica interventions to support chronic illness self-management. Jt Comm J Qual Saf 29: 563574. [Crossref]

8. Newman S, Steed L, Mulligan K (2004) Self-management interventions for chronic illness. Lancet 364: 1523-1537. [Crossref]

9. DOH (2006) Supporting people with long term conditions to self-care: A guide to developing local strategies and good practice. Leeds, UK.

10. Clark NM, Marshall H, Nancy K, Kate L, William et al. (1991) Self-management of chronic disease by older adults: A review and questions for research. J Aging Health 3: 3-27.

11. Lorig K (1996) Chronic disease self-management: A model for tertiary prevention American Behavioural Scientist 39: 676-683.

12. Lorig K (2001) Self-management in chronic illness. Key aspects of preventing and managing chronic illness, pp: 35-42.

13. Barlow J, Wright C, Sheasby J, Turner A, Hainsworth J (2002) Self-management approaches for people with chronic conditions: a review. Patient Educ Couns 48: 177187. [Crossref]

14. Loh SY, Packer T, Chinna K, Quek KF (2013) Effectiveness of a patient selfmanagement programme for breast cancer as a chronic illness: a non-randomised controlled clinical trial. J Cancer Surviv 7: 331-342. [Crossref] 
15. SY Loh, SL Chew, Boniface, Lee Luan (2011) Qualitative experiences of breast cancer survivors on a self- management intervention: 2-year post intervention focus group findings. Asia Pacific journal of cancer prevention 12: 6

16. Bodenheimer T, Wagner EH, Grumbach K (2002) Improving primary care for patients with chronic illness. JAMA 288: 1775-1779. [Crossref]

17. Von Korff M, Gruman J, Schaefer J, Curry SJ, Wagner E (1997) Collaborative Management of Chronic Illness. Annals of Internal Medicine 127: 1097-1102.

18. Siew Yim Loh, Gail Boniface, Lynette Mackenzie, Lorie Richards (2017) Professional autonomy and progress of occupational therapy - a case study on a neglected health profession in Malaysia. Journal of Hospital and Healthcare administration 1: 105.

19. SY Loh K, Khamis, PC Lee, MH Lee, KL Ow (2018) Research activity, skills and training needs of health-care professional's implications for the health education and primary health care JSM Health Education \& Primary Health Care.

20. Bultz BD, Carlson LE (2005) Emotional distress: the sixth vital sign in cancer care. $J$ Clin Oncol 23: 6440-6441. [Crossref]
21. Simonton S, Sherman A (1998) Psychological aspects of mind-body medicine: promises and pitfalls from research with cancer patients. Altern Ther Health Med 4 50-67. [Crossref]

22. Schairer C, Brown LM, Chen BE, Howard R, Lynch CF et al. (2006) Suicide after breast cancer: An international population-based study of 723,801 women. $J$ Natl Cancer Inst 98: 1416-1419. [Crossref]

23. Rampaul RS, Mullinger K, Macmillan RD, Cid J, Holmes S, et al. (2003). Incidence of clinically significant lymphoedema as a complication following surgery for primary operable breast cancer. Eur J Cancer 39: 2165-2167. [Crossref]

24. Perry S, Kowalski T, Chang CH (2007) Quality of life assessment in women with breast cancer: benefits, acceptability and utilization. Health Qual Life Outcomes5: 24. [Crossref]

25. Pusic A, Chen C, Cano S, Klassen A, McCarthy C, et al. (2007) measuring quality of life in cosmetic and reconstructive breast surgery: A systematic review of patientreported outcomes instruments. Plast Reconstr Surg 120: 823-837. [Crossref]

Copyright: $\odot 2018$ Loh SY. This is an open-access article distributed under the terms of the Creative Commons Attribution License, which permits unrestricted use, distribution, and reproduction in any medium, provided the original author and source are credited. 